\title{
Searches for heavy resonances decaying to top quarks with the ATLAS detector
}

Farida Fassi ${ }^{1}$, on behalf of the ATLAS collaboration

University Mohammed V in Rabat, Faculty of Sciences

4 av. Ibn Battouta, B.P. 1014, R.P. 10000, 14, Morocco

E-mail:farida.fassi@cern.ch

\begin{abstract}
Searches for new resonances that decay either to pairs of top quarks or a top and a b-quark will be presented. The searches are performed with the ATLAS experiment at the LHC using proton-proton collision data collected at a centre-of-mass energy of $13 \mathrm{TeV}$. The invariant mass spectrum of hypothetical resonances are examined for local excesses or deficits that are inconsistent with the Standard Model prediction. No significant deviation from the prediction is found so far.
\end{abstract}

39th International Conference on High Energy Physics (ICHEP2018),

July 4-11, 2018

Seoul, South Korea,

\section{${ }^{1}$ Speaker}




\section{Introduction}

The top quark is a constituent of matter with unique characteristics. Its mass is the largest one amongst the fundamental fermions and it is intriguinly close to the scale of the electronweek symmetry breaking (EWSB). As such, it plays a special role in many Beyond the Standard Model (BSM) theories of EWSB. In models with top condensation such as technicolor and topcolor the role of the SM Higgs bosons is filled by a composite particle that is a $t \bar{t}$ bound state [1]. These models predict additional heavy gauge bosons, which couple strongly to top quarks, e.g. colorsinglet $Z^{\prime}$ [2], color octets, such as colorons or axigluons. Further models are two Higgs doublet and Minimal Supersymmetric models, where pseudoscalar $(A)$ and scalar $(H)$ Higgs bosons may couple strongly to top quarks. The weakness of gravity compared to other forces (hierarchy problem) has been addressed in the context of extra dimesnsion, such as the Rundall-Sundrum and ADD models. In many of these models gauge interations exist whose couplings with the third generation quarks and in particular the top quark are enhanced. Interference effects between the signal process and SM $t \bar{t}$ production, which are expected to distort the signal shape [3] from a single peak to a peak-dip structure, are taken into account in this contribution.

This proceedings presents, in a way that is model-independents as much as possible, the experimental sensitivity in ATLAS to new resonances in the $t \bar{t}$ invariant mass distribution. This is done in a scenario of the integrated luminosity of $36.1 \mathrm{fb}^{-1}$ collected by the ATLAS detector from LHC proton-proton collisions at a center-of-mass energy of $\sqrt{s}_{\mathrm{s}}=13 \mathrm{TeV}$ in 2015 and 2016.

\section{Search for $\mathrm{t}_{\bar{t}}$ resonances in the lepton+jets final state}

The search for new particles in the $t \bar{t}$ production is performed [4] in the lepton+jets topology, using a single muon or electron (indicated with $\ell$ ) as a trigger. The signature is a deviation from the $t \bar{t}$ invariant mass spectrum predicted by the SM. The selection requires a single isolated $\ell$ with $p_{\mathrm{T}}>25 \mathrm{GeV}$, large missing transverse momentum $\left(E_{\mathrm{T}}^{\text {miss }}\right)$, and hadronic jets. At least one of the jets must be identified as a $b$-jet. The $b$-tagged track-jets are used to categorise the accepted events into several channels. Based on the hadronic activity, the event is classified as Boosted or Resolved. The reconstruction of the event is based on three types of jets, all using the anti- $k_{\mathrm{t}}$ algorithm: the small- $R$ jets are the standard, calorimeter-based jets, reconstructed with a radius parameter $R=0.4$; with $p_{\mathrm{T}}>25 \mathrm{GeV}$ and $|\eta|<2.5$. The large- $R$ jets, with $p_{\mathrm{T}}>300 \mathrm{GeV}$ and $|\eta|<$ 2.0 , are reconstructed with $R=1.0$, but also have a "trimming" process, to discard low-energy sub-jet components, which are due to event pile-up. The track-jets are reconstructed from charged tracks, with $R=0.2, p_{\mathrm{T}}>10 \mathrm{GeV}$ and $|\eta|<2.5$. A lepton $p_{\mathrm{T}}$-dependent isolation cut is also used. An event passes the Boosted selection if meets the following criteria: A small-R jet (Jsel), with no specific $b$-tagging is required to be near to the lepton, at $\Delta R($ jet- $\ell)<1.5\left(\Delta R=\sqrt{\Delta \eta^{2}+\Delta \varphi^{2}}\right)$. The large- $R$ jet is required to be at a large azimuthal angle from both the lepton $\left(\Delta \varphi_{1}>2.3 \mathrm{rad}\right)$ and from $J_{s e l}\left(\Delta \varphi_{2}>1.5 \mathrm{rad}\right)$. Events that fail any of these Boosted selection requirements are classified as passing the Resolved selection if there are at least 4 small- $R$ jets with $p_{\mathrm{T}}>25 \mathrm{GeV}$ and if the $\chi^{2}$ algorithm for reconstructing $t \bar{t}$ system [10] yields a value of $\log _{10}\left(\chi^{2}\right)<0.9$. Cuts on $E_{\mathrm{T}}^{\text {miss }}$ are also required: $E_{\mathrm{T}}^{\text {miss }}>20 \mathrm{GeV}$ and $E_{\mathrm{T}}^{\text {miss }}+m_{T}^{W}>60 \mathrm{GeV}$. The $t \bar{t}$ system is reconstructed from both kinematic regimes, Boosted and Resolved. The simulation agrees well with the data, within the systematic uncertainties, and a statistical fit is performed to the invariant $t \bar{t}$ mass spectra (as shown in Fig.1 left) using the BumpHunter algorithm, with nuisance parameter profile fit. The main systematic uncertainties are related to the large- $R$ jet energy scale. From the fit, using 
various hypotheses for the mass and width, it is possible to establish limits on the cross section times the branching ratio to top quark pairs.
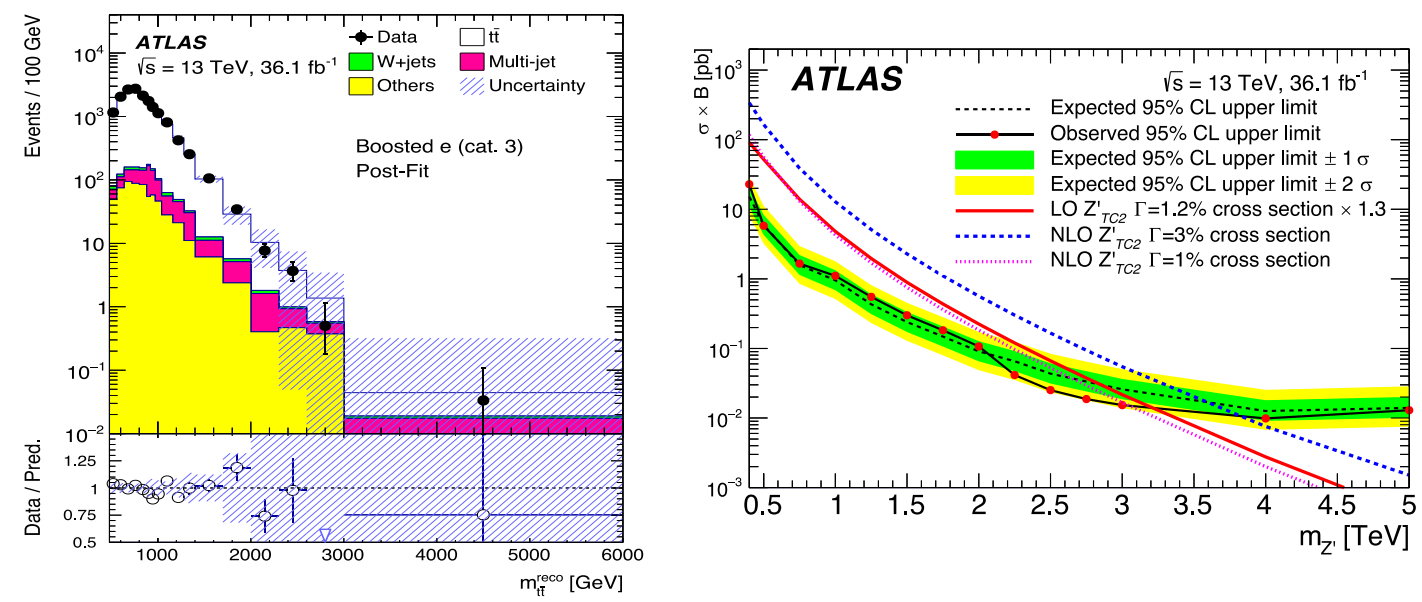

Fig 1. The $t \bar{t}$ invariant mass spectra for Boosted analyses (left panel), and observed and expected upper limits (right panel) at 95\% CL of $Z^{\prime}$ Reference [4].

The sensitivity of the search is tested new colour-singlet/octet bosons with spin $1 / 2$ and masses from 0.4 to $5 \mathrm{TeV}$. The resulting constraints on the mass at 95\%C.L.; a Topcolour-assisted Technicolour, $\mathrm{Z}_{\mathrm{TC} 2}^{\prime}$, with a width of $1 \%$ is excluded for masses $\mathrm{Z}_{\mathrm{TC} 2}^{\prime}<3.0 \mathrm{TeV}$. An axial-vector mediator, $\mathrm{Z}_{\mathrm{DM}, \mathrm{ax}}^{\prime}$ arising in simplified models of dark matter is excluded for masses $\mathrm{Z}_{\mathrm{DM}, \mathrm{ax}}^{\prime}<1.4$ $\mathrm{TeV}$. The search allowed to exclude at 95\% C.L. Kaluza-Klein (KK) gravitons in the range 0.45 $\mathrm{TeV}<m_{G_{K K}}<0.65 \mathrm{TeV}$ (Fig. 2, right), a KK gluon of width $30 \%$ for $m_{g_{K K}}<3.7 \mathrm{TeV}$, while a KK gluon of width $15 \%$ is excluded for $m_{g_{K K}}<3.8 \mathrm{TeV}$ as shown in Fig. 2 (left).
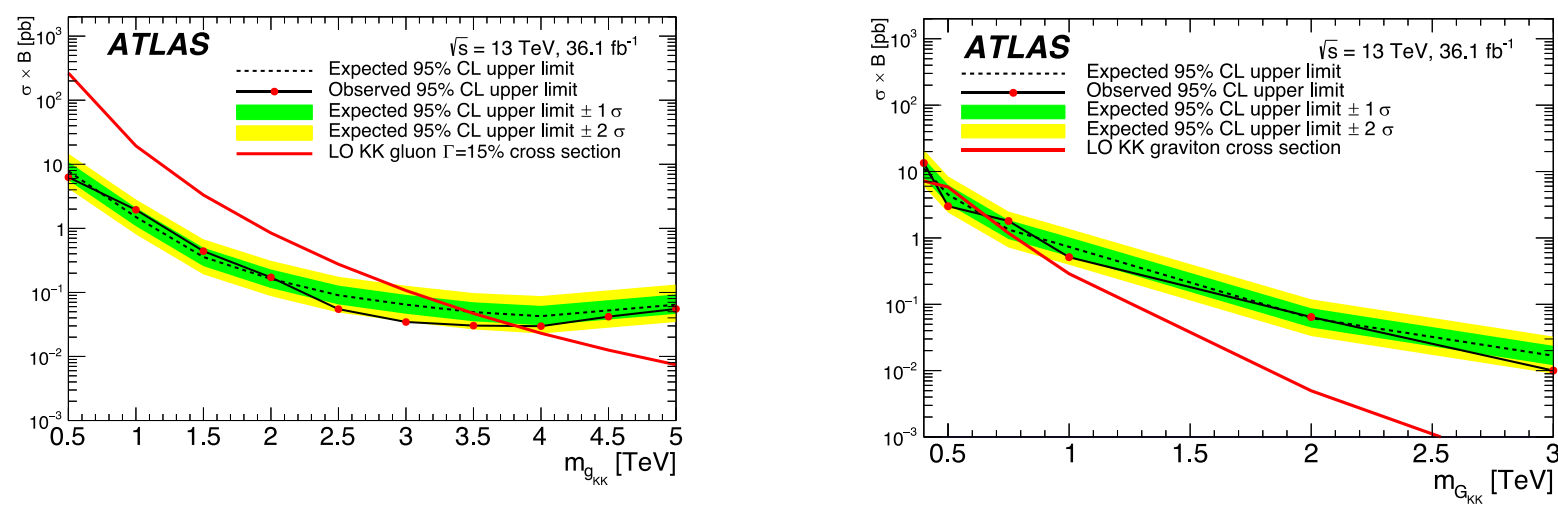

Fig 2. Observed and expected upper limits at $95 \% \mathrm{C} \mathrm{L}$ on the production times branching ratio of the KK gluon (left panel), and KK graviton (right panel) [4].

\section{Search for Heavy Higgs Bosons $\mathrm{A} / \mathrm{H}$ in $\mathrm{t} \bar{t}$ production}

A search for heavy pseudoscalar $(A)$ and scalar $(H)$ Higgs bosons decaying into $t \bar{t}$ is perfomed using $20.3 \mathrm{fb}^{-1}$ of pp collision data at $\sqrt{\mathrm{s}}_{\mathrm{s}}=8 \mathrm{TeV}[5]$. Interference between the gluon-gluon initiated loop production and the SM $t \bar{t}$ production yields a non-negligible imaginary term in the 
amplitude. As a result of the interference, the signal shape is distorted from a Breit-Wigner peak to a peak-dip structure. The search used the Resolved regime jet configuration in the $\ell+$ jets channel, requiring at least 4 small- $R$ jets, each with $p_{\mathrm{T}}>25 \mathrm{GeV}$, and at least one $b$-tagged jet with $70 \%$ efficiency, with the same requirements on leptons and $E_{\mathrm{T}}^{\mathrm{miss}}$ as in the Boosted analysis (see Sect. 2.). No significant deviation from the Standard Model prediction is observed in the $t \bar{t}$ invariant mass spectrum. The results are interpreted in terms of a CP-conserving $2 \mathrm{HDM}$ of type II, with a softly broken $Z_{2}$ symmetry. The lighter of the two neutral CP-even states, $h$, is assumed to be the Higgs boson, with a mass of $m_{\mathrm{h}}=125 \mathrm{GeV}[6]$ and couplings as predicted by the SM. The exclusion limits are derived separately for each signal hypothesis from a profile-likelihood fit. The upper limits on signal strength at $95 \% \mathrm{CL}$ are obtained with the CLs method for a grid of values in the $\left(m_{\mathrm{A} / \mathrm{H}}, \tan \beta\right)$ plane, as shown in Fig 3.

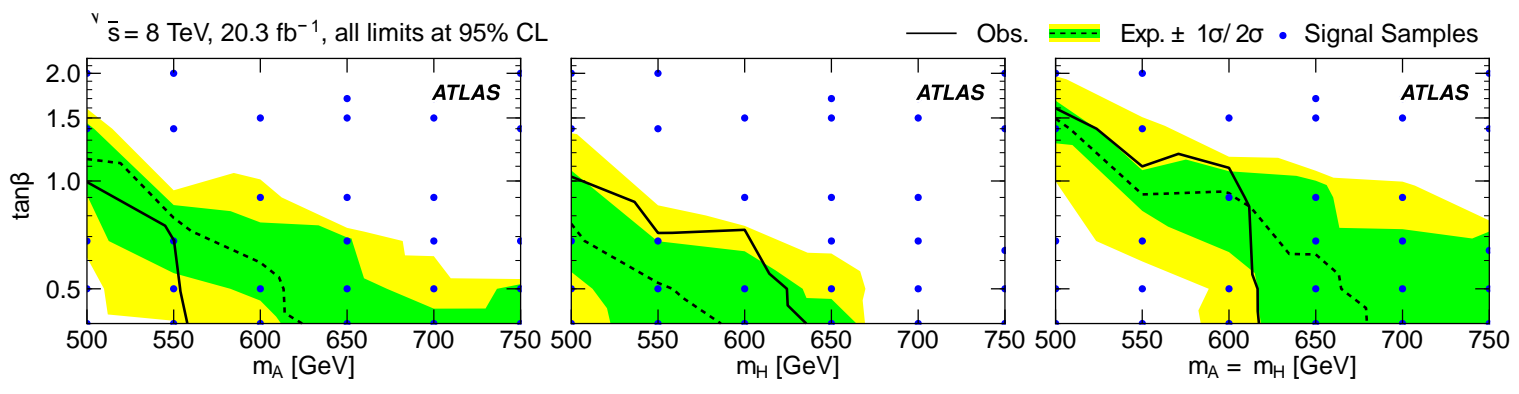

Fig 3. Exclusion limits in the $\tan \beta$-mass plane for the scalar and pseudoscalar hypotheses

\section{Conclusion}

The most recent results on searches for new physics beyond the SM were presented using 36.1 $\mathrm{fb}^{-1}$ of $13 \mathrm{TeV}$ pp collision data collected by the ATLAS detector at the LHC in 2015 and 2016. No deviations from the SM are observed and limits are derived on the production rate times branching fraction in various models were derived at $95 \% \mathrm{CL}$. Previously placed ATLAS exclusions could be strongly improved and in many cases the most stringent limits to date are provided. Considered are heavy vector gauge bosons, technicolor, technicolor simplified models of dark matter, graviton and gluon in the Randall-Sundrum ADD models and 2HDM-II model.

\section{References}

[1] G. Cvetic, Reviews of Modern Physics 71,513 (1999).

[2] J. Rosner, Phys. Lett. B387, 113 (1996); M. Carena et al., Phys. Rev. D 70, 093009 (2004)

[3] R. Frederix and F. Maltoni, JHEP 0901, 047 (2009).

[4] ATLAS Collaboration, Search for heavy particles decaying into top-quark pairs using lepton-plusjets events in proton-proton collisions at $\sqrt{s}=13 \mathrm{TeV}$ with the ATLAS detector. Eur. Phys. J. C 78 (2018) 565

[5] ATLAS collaborateon, Search for a heavy pseudo-scalar/scalar particle decaying to a top quark pair in pp collisions at $\sqrt{\mathrm{s}}_{\mathrm{s}}=8 \mathrm{TeV}$ with the ATLAS detector, arXiv: 1707.06025

[6] ATLAS collaboration, Observation of a new particle in the search for the Standard Model Higgs boson with the ATLAS detector at the LHC, Physics Letters B, vol. 716 n. 1 p. 1 (2012). 